Supporting Information

\title{
Sodium-ion Storage Mechanism in Triquinoxalinylene and Strategy for Improving Electrode Stability
}

Qinglan Zhao , Wei Zhao ${ }^{b}$, Cheng Zhang, Yilan Wu ${ }^{a}$, Qinghong Yuan ${ }^{d}$, Andrew K. Whittaker ${ }^{c}$, and X. S. Zhao $a^{*}$

${ }^{\text {a }}$ School of Chemical Engineering, The University of Queensland, Brisbane, QLD 4072, Australia

${ }^{\mathrm{b}}$ State Key Laboratory of Precision Spectroscopy, School of Physics and Electronic Science, East China Normal University, 3663 N. Zhongshan Road, Shanghai 200062, China

${ }^{\mathrm{c}}$ Australian Institute for Bioengineering and Nanotechnology and ARC Centre of Excellence in Convergent Bio-Nano Science and Technology, The University of Queensland, Brisbane, QLD 4072, Australia

${ }^{d}$ Centre for Theoretical and Computational Molecular Science, Australian Institute for Bioengineering and Nanotechnology, The University of Queensland, Brisbane, QLD, 4072, Australia

*E-mail: george.zhao@uq.edu.au 


\section{Experimental Section}

\section{Synthesis of Triquinoxalinylene}

Triquinoxalinylene (TQA) was synthesized by the condensation reaction of hexaketocyclohexane and o-phenylenediamine. ${ }^{\text {Error! Reference source not found. Typically, } 1.06 \mathrm{~g} \text { o- }}$ phenylenediamine and $1.031 \mathrm{~g}$ hexaketocyclohexane were mixed in $50 \mathrm{~mL}$ acetic acid/ethanol (1:1 in vol./vol.) saturated with argon. After reflux for $24 \mathrm{~h}$, a dark green suspension was centrifuged and washed with acetic acid, acetone, water and ethanol, followed by boiling in $50 \mathrm{ml} 30 \mathrm{wt} \% \mathrm{HNO}_{3}$ at $140{ }^{\circ} \mathrm{C}$ for $3 \mathrm{~h}$. Then, a brown suspension was centrifuged, washed using deionized water and ethanol, and dried under vacuum to obtain a yellow powder. ${ }^{1} \mathrm{H}$ NMR (400 MHz, $\left.\mathrm{CF}_{3} \mathrm{COOH}, \mathrm{D}_{2} \mathrm{O}, 300 \mathrm{~K}\right): \delta(\mathrm{ppm}) 8.7$ and 8.4; ${ }^{13} \mathrm{C} \mathrm{NMR}(125 \mathrm{MHz}$, $\left.\mathrm{CF}_{3} \mathrm{COOH}, \mathrm{D}_{2} \mathrm{O}, 300 \mathrm{~K}\right): \delta(\mathrm{ppm}) 141.8,139.2,136.7$ and 128.2. Solution ${ }^{1} \mathrm{H} \mathrm{NMR},{ }^{13} \mathrm{C}$ NMR, ${ }^{1} \mathrm{H}_{-}{ }^{13} \mathrm{C} \mathrm{HSQC}$ and ${ }^{1} \mathrm{H}_{-}{ }^{13} \mathrm{C}$ HMBC spectra were recorded on a Bruker Avance $400 \mathrm{MHz}$ spectrometer.

${ }^{1} \mathrm{H}-{ }^{13} \mathrm{C}$ HSQC spectra were acquired with a relaxation delay of $1.5 \mathrm{~s}$ and spectral widths of $5342 \mathrm{~Hz}$ in the ${ }^{1} \mathrm{H}$ dimension and $22136 \mathrm{~Hz}$ in the ${ }^{13} \mathrm{C}$ dimension. 16 scans were collected. The size of fid of $F_{1}$ and $F_{2}$ was 256 and 2048 points. $90^{\circ}$ pulse width was $8.07 \mu$ s, and the power level for pulse was $-9.82 \mathrm{dBW}$. The QSINE was used as the window function with sine bell shift at 2 .

${ }^{1} \mathrm{H}-{ }^{13} \mathrm{C}$ HMBC spectra were acquired with a relaxation delay of $1.5 \mathrm{~s}$ and spectral widths of $4808 \mathrm{~Hz}$ in the ${ }^{1} \mathrm{H}$ dimension and $22321 \mathrm{~Hz}$ in the ${ }^{13} \mathrm{C}$ dimension. 16 scans were collected. The size of fid of $F_{1}$ and $F_{2}$ was 128 and 2048 points. $90^{\circ}$ pulse width was $8.07 \mu$ s, and the power level for pulse was $-9.82 \mathrm{dBW}$. The SINE was used as the window function without sine bell shift. 


\section{Electrochemical Measurements}

A slurry of $30 \mathrm{mg}$ TQA, $15 \mathrm{mg}$ carbon black and $5 \mathrm{mg}$ poly(vinylidene difluoride) (PVDF) binder in appropriate amount of N-methyl pyrrolidinone (NMP), unless otherwise noted, were casted on the copper foil to make the working electrode without Nafion. For the modified electrode, $500 \mu \mathrm{L} 5 \mathrm{wt} \%$ Nafion solution was added during the slurry preparation followed by vigorous stirring and sonication. After drying the electrode, another $100 \mu \mathrm{L} 0.5$ wt\% Nafion solution was dropped on the surface of the electrode to make a top thin Nafion membrane. The Nafion in the electrode was sodiated by treating the as-prepared electrode with $0.1 \mathrm{~mol} \mathrm{~L}^{-1} \mathrm{NaOH}$ followed by rinsing and drying. Sodium foil was employed as the counter electrode, glass microfiber (Whatman GF/D, Aldrich) was used as the separator, and $1 \mathrm{~mol} \mathrm{~L}^{-1} \mathrm{NaPF}_{6}$ in diglyme was applied as the electrolyte.

All cells were assembled in a glove box with the contents of oxygen and water less than 0.1 ppm. The cyclic voltammetry $(\mathrm{CV})$ curves in the potential range of $0.1-2.5 \mathrm{~V}\left(v s . \mathrm{Na} / \mathrm{Na}^{+}\right)$ were recorded on a multichannel potentiostat (VMP3, BioLogic Science Instruments). The galvanostatic discharge-charge tests were performed on a battery cycler (CT3008, Neware).

\section{Electrode Sample Preparation for Magnetic Resonance Spectroscopy}

Prototype pouch cells using a sodium foil as the counter electrode, a glass microfiber (Whatman GF/D, Aldrich) as the separator and $1 \mathrm{~mol} \mathrm{~L}^{-1} \mathrm{NaClO}_{4}$ in diglyme as the electrolyte were designed to obtain sufficient samples for magnetic resonance signals. The ratio of $6: 3: 1$ for TQA, carbon black and PVDF was used to make the working electrode for pouch cells. The working electrode was collected from the disassembled pouch cell until a desired state of charge under a 30 min floating, washed with dimethyl carbonate (DMC), and dried under 
vacuum. The samples were scratched from the electrode and packed in a $4 \mathrm{~mm}$ magic angle spinning (MAS) probe for nuclear magnetic resonance (NMR) measurements or packed in a quartz tube for electron paramagnetic resonance (EPR) tests in the glove box.

\section{Solid-State ${ }^{13} \mathrm{C}$ and ${ }^{23} \mathrm{Na}$ MAS NMR Spectroscopy}

${ }^{13} \mathrm{C}$ and ${ }^{23} \mathrm{Na}$ MAS NMR spectra were recorded on a Bruker Avance III $300 \mathrm{MHz}$ spectrometer operating at a ${ }^{13} \mathrm{C}$ frequency of $75.468 \mathrm{MHz}$ and a ${ }^{23} \mathrm{Na}$ frequency of 132.295 $\mathrm{MHz}$ under a rotor spinning rate of 5 or $6 \mathrm{kHz}$. The ${ }^{23} \mathrm{Na}$ chemical shift was referenced to 1 mol L ${ }^{-1} \mathrm{NaCl}$ aqueous solution. Deconvolution of NMR spectra was done using the PeakFit software.

\section{EPR Spectroscopy}

EPR spectra were measured on a Bruker Elexsys E500 CW (Q-, X-, S-band) variable temperature (1.5-400K) EPR spectrometer operating at $9.206 \mathrm{GHz}$ with a field modulation frequency of $100 \mathrm{kHz}$. The EPR acquisition was performed at a microwave power of $1 \mathrm{~mW}$ and a modulation amplitude of $25 \mathrm{mT}$.

\section{Density Functional Theory Calculation}

All calculations were performed with Gaussian 09. The hybrid density functional (B3LYP) and a 6-31G $(\mathrm{d}, \mathrm{p})$ basis set were applied to calculate the Gaussian optimized geometries for the molecules. Tetrahydrofuran with similar physical properties to diglyme was used as the solvent for the polarization continuum model (PCM). The thermal correction and the Gibbs free energy were obtained from vibrational frequency calculations in the solvent.

The binding energy ( $\Delta E$, hartree) of the molecules during the sodiation process was calculated using the equation as follows: 


$$
\Delta E=E_{n N a}-\left(E_{(n-1) N a}+E_{N a(0)}\right)
$$

where $\mathrm{n}$ is the number of sodium atoms, and $E_{n N a}, E_{(n-1) N a}$ and $E_{N a(0)}$ are the sum of electronic and zero-point energies for a sodiated molecule with $\mathrm{n}$ sodium atoms, $\mathrm{n}-1$ sodium atoms and the sodium atom, respectively.

The redox potential $\left(\boldsymbol{E}_{\text {redox }}^{\boldsymbol{o}}, \mathrm{V}\right)$ of the sodiation process was calculated using the following equation:

$$
E_{\text {redox }}^{o}=-\left(G_{f}^{o}-G_{i}^{o}-G_{N a}^{o}\right) / n F
$$

where $\boldsymbol{G}_{\boldsymbol{f}}^{\boldsymbol{o}}, \boldsymbol{G}_{\boldsymbol{i}}^{\boldsymbol{o}}$ and $\boldsymbol{G}_{\boldsymbol{N} \boldsymbol{a}}^{\boldsymbol{o}}\left(\mathrm{kcal} \mathrm{mole}^{-1}\right)$ are the sum of electronic and thermal Gibbs free energy for a molecule at the final and the initial sodiated states, and the sodium atom; $\mathrm{n}$ is the number of electrons during sodiation, and $F$ is the Faraday constant. 

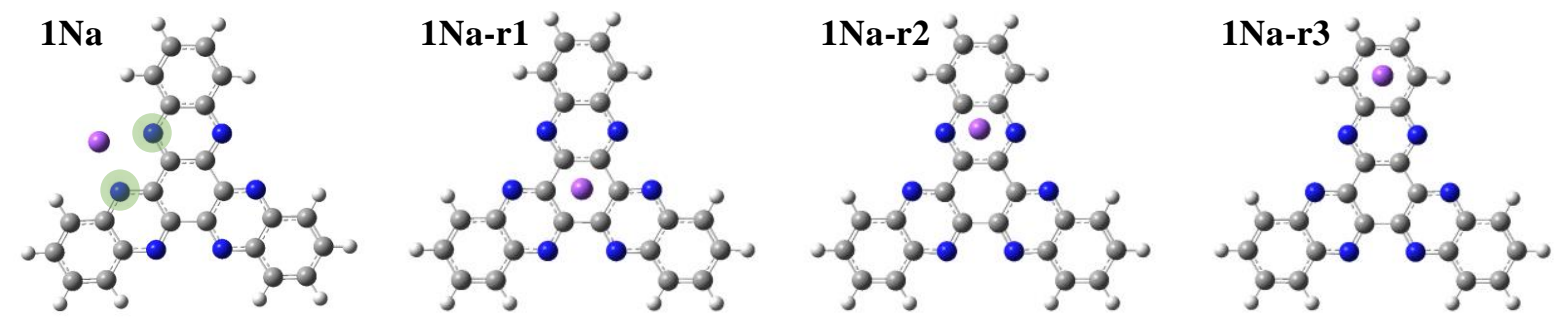

○

○

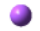

.000000000000

Figure S1. The structures for the coordination of TQA molecule with the first sodium ion simulated by density functional theory (DFT) with the side views. (The green area indicates the two bidentate nitrogens interacted with the sodium ion.)

Table S1. The electronic and zero-point energies along with the binding energies $(\Delta \mathrm{E})$ calculated for the coordination of TQA molecule with the first sodium ion. $\mathrm{E}_{\mathrm{Na}(0)}=-162.2950$ hartree $=-0.4416\left(\mathrm{eV} \times 10^{4}\right)$.

\begin{tabular}{ccccccc}
\hline & & TQA & $1 \mathrm{Na}$ & $1 \mathrm{Na}-\mathrm{r} 1$ & $1 \mathrm{Na}-\mathrm{r} 2$ & 1Na-r3 \\
\hline Energy & $($ hartree $)$ & -1250.0856 & -1412.4347 & -1412.3842 & -1412.3924 & -1412.3892 \\
& $\left(\mathrm{eV} \times 10^{4}\right)$ & -3.4015 & -3.8432 & -3.8431 & -3.8431 & -3.8431 \\
$\Delta \mathrm{E}=\mathrm{E}_{\mathrm{Na}^{-}}$ & $($hartree $)$ & & -0.0541 & -0.0036 & -0.0118 & -0.0086 \\
$\left(\mathrm{E}_{\mathrm{TQA}}+\mathrm{E}_{\mathrm{Na}(0)}\right)$ & $\left(\mathrm{eV} \times 10^{4}\right)$ & & -1.47 & -0.10 & -0.32 & -0.23 \\
\hline
\end{tabular}



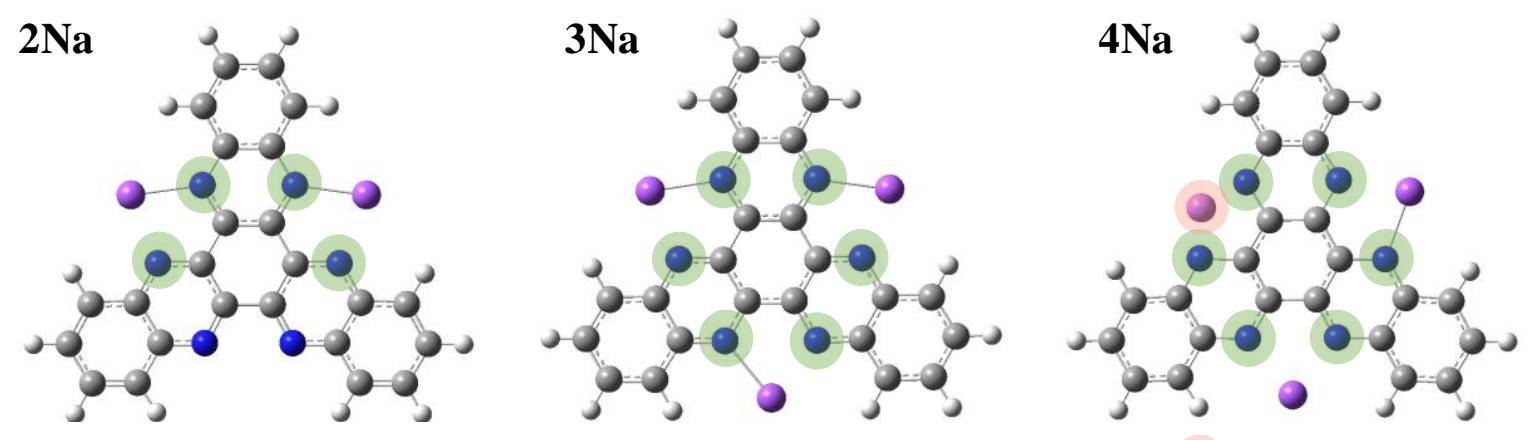

100000000000
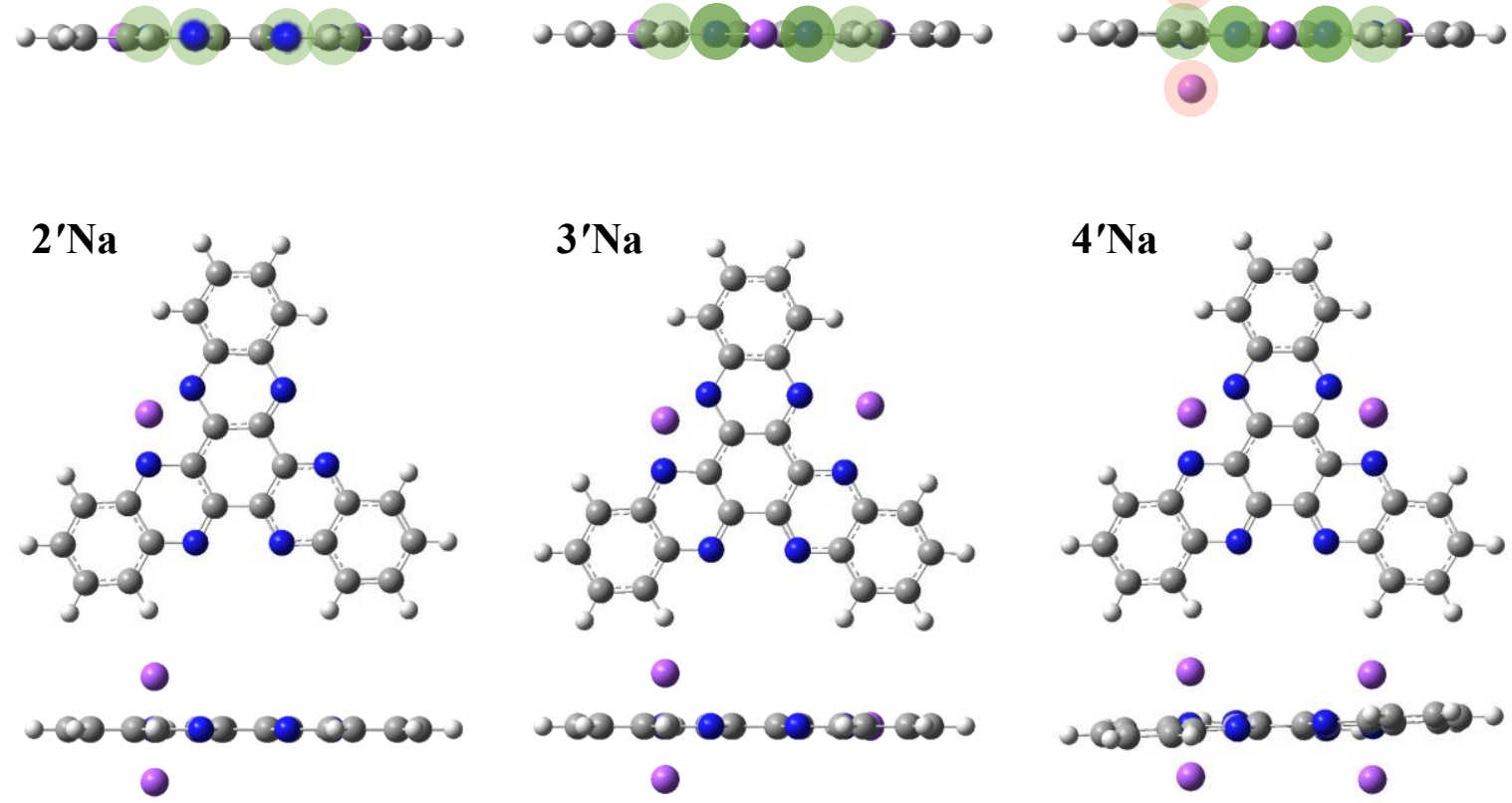

$\Delta E=E_{n^{\prime} \mathrm{Na}}-E_{n N a}=+0.9 \mathrm{eV}$

$+0.93 \mathrm{eV}$

$+0.89 \mathrm{eV}$

Figure S2. Comparison among the structures for the coordination of TQA molecule with two, three and four sodium ions simulated by DFT with the side views. (The green area indicates the two bidentate nitrogens interacting with the sodium ions, and the pink area indicates the sodium ions out of the plane.) 
$1 \mathrm{Na}$<smiles>CCOC(=O)CC</smiles>

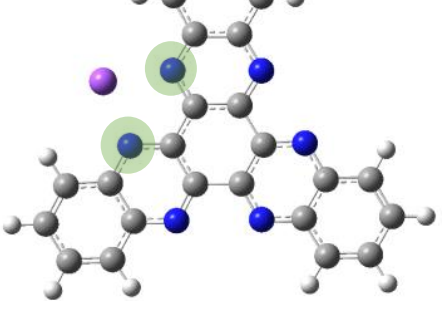

$. \infty 0000-\infty 000$
$2 \mathrm{Na}$

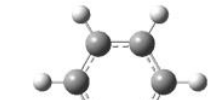<smiles>[C]1CCCC1</smiles>

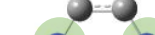

10

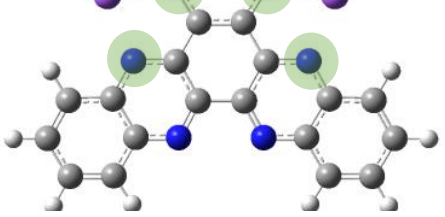

.00000000000

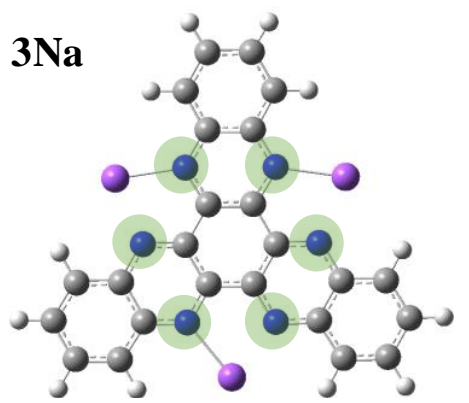

.0000000000
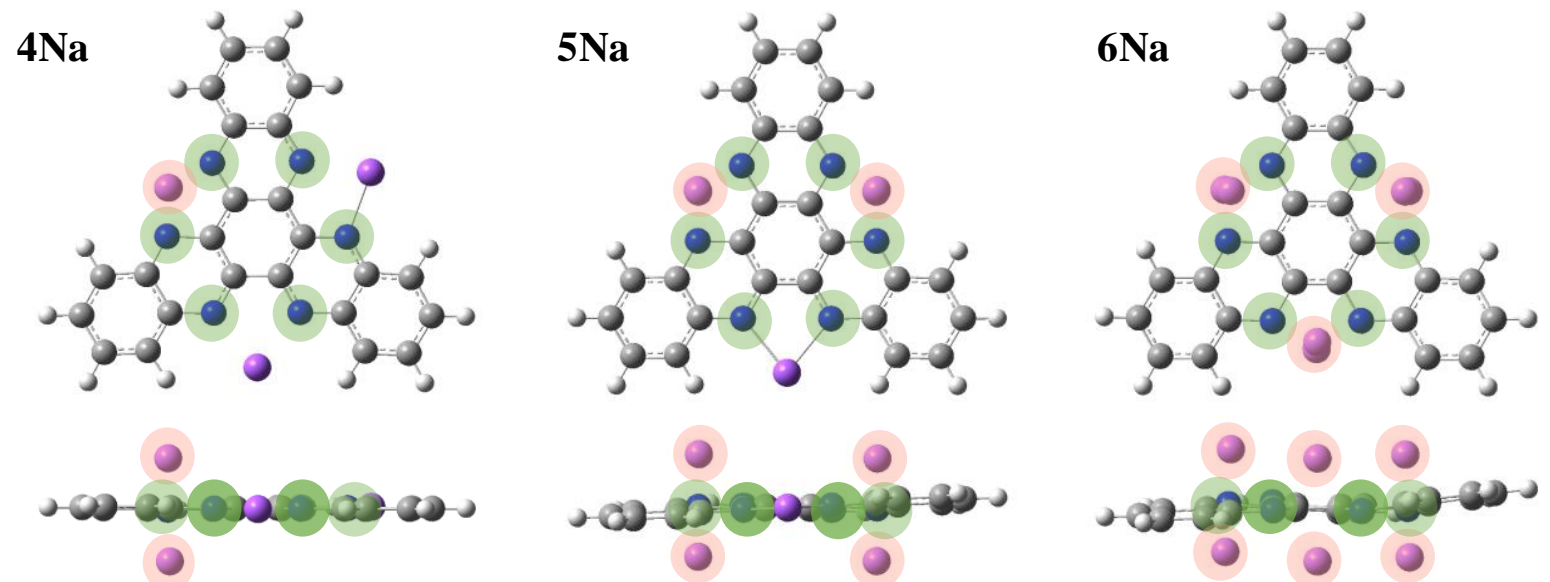

Figure S3. The optimized structure of the coordination between the TQA molecule and six sodium ions with the side views. (The green area indicates the two bidentate nitrogens interacted with the sodium ion, and the pink area indicates the sodium ions out of the plane.) 
Table S2. Sum of the electronic and thermal Gibbs free energies with the binding energies $(\triangle \mathrm{E})\left(\mathrm{eV} \times 10^{4}\right)$ for the optimized coordination of TQA molecule with six sodium ions, and the redox potentials $(\mathrm{V})$ calculated at the B3LYP/6-31+G(d,p) level in THF solvent. $\mathrm{E}_{\mathrm{Na}(0)}=$ $-0.4416\left(\mathrm{eV} \times 10^{4}\right)$

\begin{tabular}{cccccccc}
\hline & TQA & $1 \mathrm{Na}$ & $2 \mathrm{Na}$ & $3 \mathrm{Na}$ & $4 \mathrm{Na}$ & $5 \mathrm{Na}$ & $6 \mathrm{Na}$ \\
& $(0 \mathrm{Na})$ & & & & & & \\
\hline Energy & -3.4015 & -3.8432 & -4.2850 & -4.7267 & -5.1683 & -5.6100 & -6.0516 \\
$\begin{array}{c}\Delta \mathrm{E}=\mathrm{E}_{\mathrm{nNa}}{ }^{-} \\
\left(\mathrm{E}_{(\mathrm{n}-1) \mathrm{Na}+\mathrm{E}}+\mathrm{E}_{\mathrm{Na}(0)}\right)\end{array}$ & & -1.47 & -1.34 & -1.36 & -0.18 & -0.45 & -0.29 \\
\hline
\end{tabular}



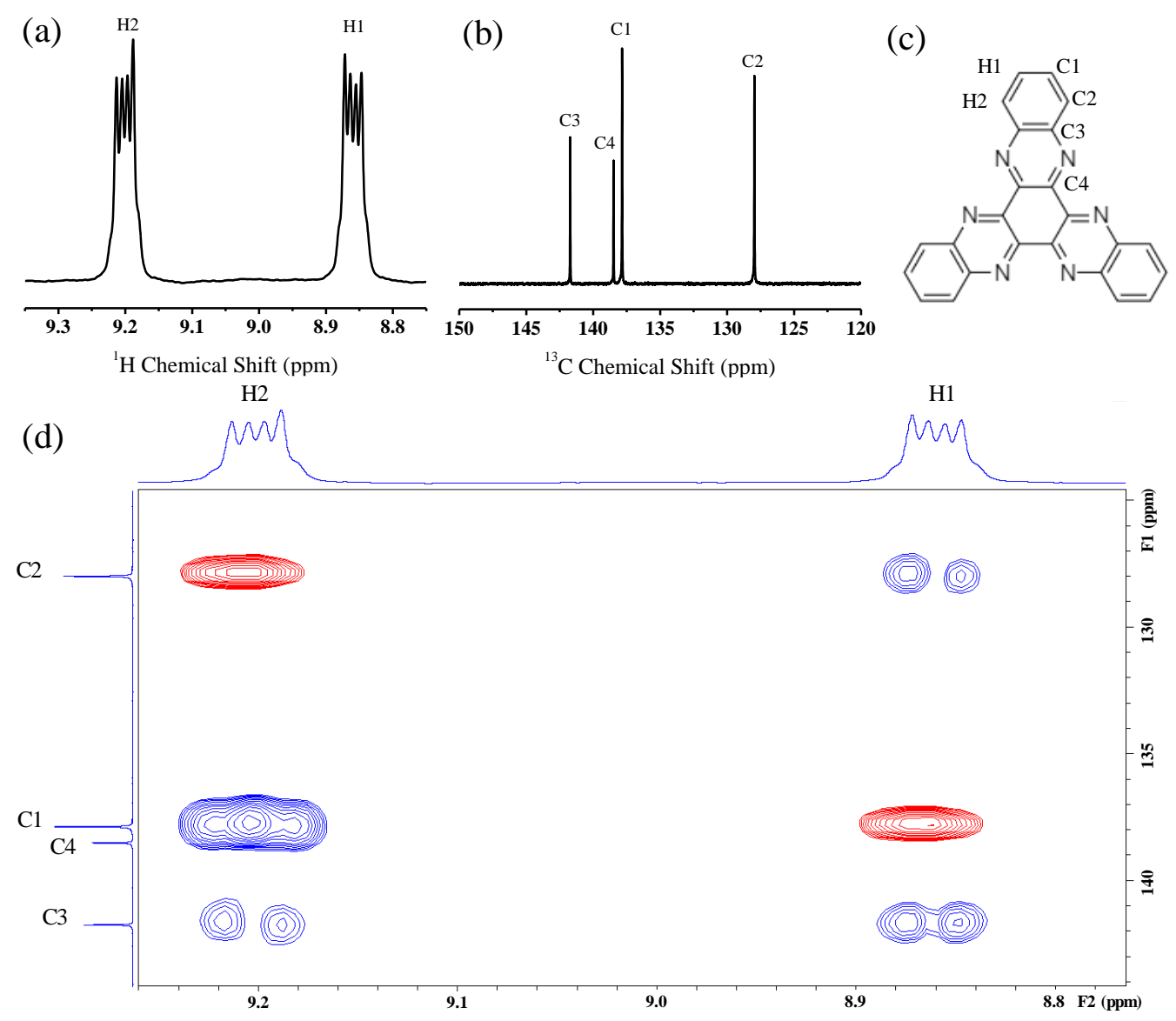

Figure S4. (a) $1 \mathrm{D}{ }^{1} \mathrm{H}$ NMR spectrum $\left(400 \mathrm{MHz}, \mathrm{CF}_{3} \mathrm{COOH}, \mathrm{D}_{2} \mathrm{O}, 300 \mathrm{~K}\right.$ ), (b) ${ }^{13} \mathrm{C} \mathrm{NMR}$ spectrum $\left(125 \mathrm{MHz}, \mathrm{CF}_{3} \mathrm{COOH}, \mathrm{D}_{2} \mathrm{O}, 300 \mathrm{~K}\right)$, (c) molecular structure of TQA with assignments, and (d) $2 \mathrm{D}^{1} \mathrm{H}_{-}{ }^{13} \mathrm{C} \mathrm{HSQC}$ (red) and ${ }^{1} \mathrm{H}-{ }^{13} \mathrm{C} \mathrm{HMBC}$ (blue) spectra. 
Table S3. The number of sodium ions stored per TQA molecule at a certain potential (V vs. $\mathrm{Na} / \mathrm{Na}^{+}$) obtained from the $2^{\text {nd }}$ discharge by subtracting the contribution from the carbon black/PVDF component.

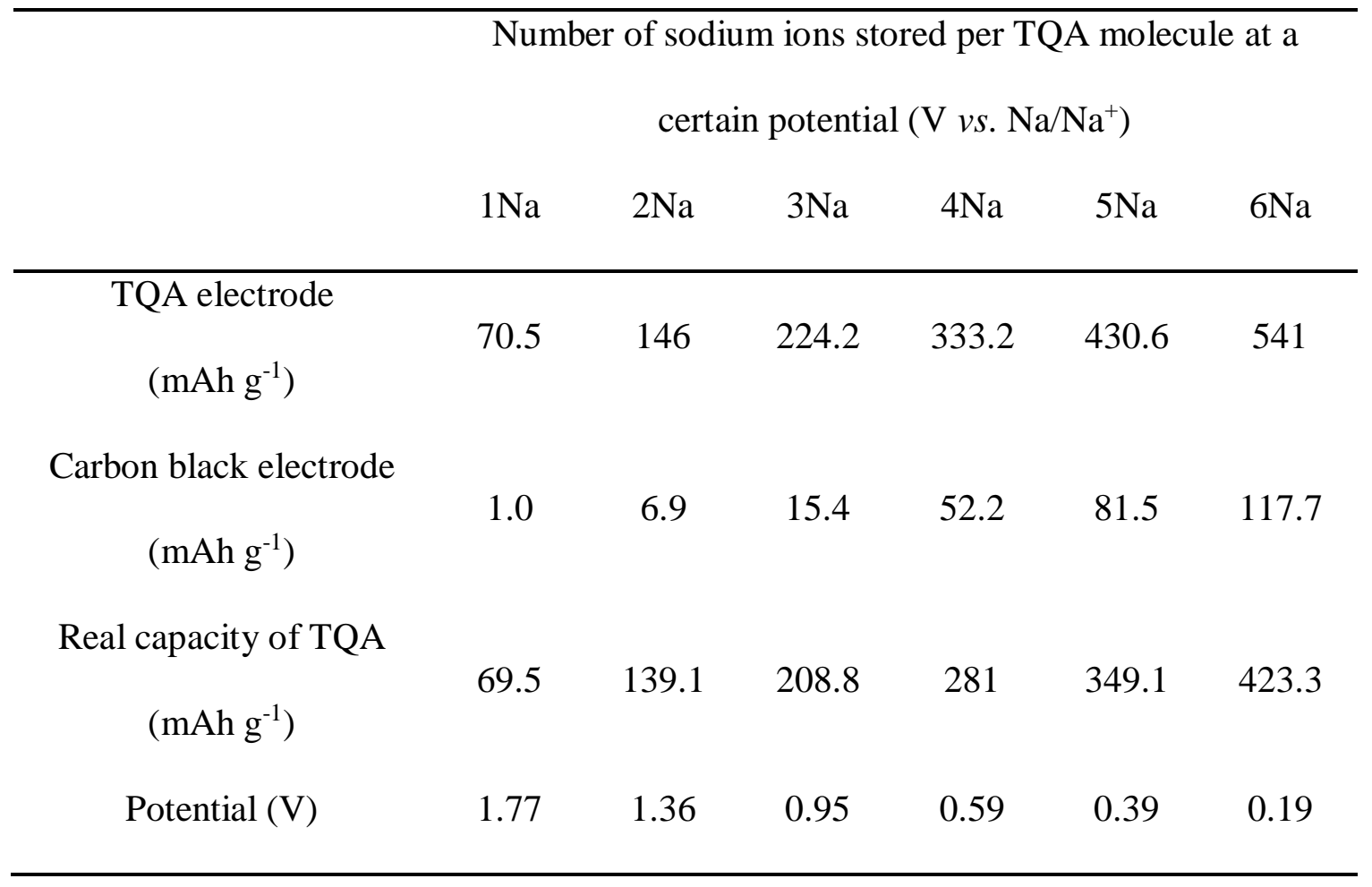

The theoretical capacity of TQA was calculated to be $419 \mathrm{mAh} \mathrm{g}^{-1}$ on the basis of the sixelectron transfer mechanism using the Equation (1): ${ }^{(1)}$

$C\left(\mathrm{mAhg}^{-1}\right)=\frac{n \times F\left(\mathrm{Cmol}^{-1}\right)}{M_{w}\left(\mathrm{gmol}^{-1}\right)}=\frac{n \times 96485(\mathrm{C})}{M_{w}(\mathrm{~g})}=\frac{n \times 96485(\mathrm{As})}{M_{w}(\mathrm{~g})}=\frac{n \times 96485 \times 1000 / 3600(\mathrm{mAh})}{M_{w}(\mathrm{~g})}=\frac{26801 \times n}{M_{w}}\left(\mathrm{mAhg}^{-1}\right)$

where, $C, n, F$ and $M_{w}$ are the theoretical specific capacity, the transferred electron number in each formula unit (here $n=6$ ), the Faraday constant and the molecular weight of the formula unit, respectively. The capacity contributed from one sodium-ion storage is 69.8 $\mathrm{mAh} \mathrm{g}^{-1}$, according to the equation (1). 


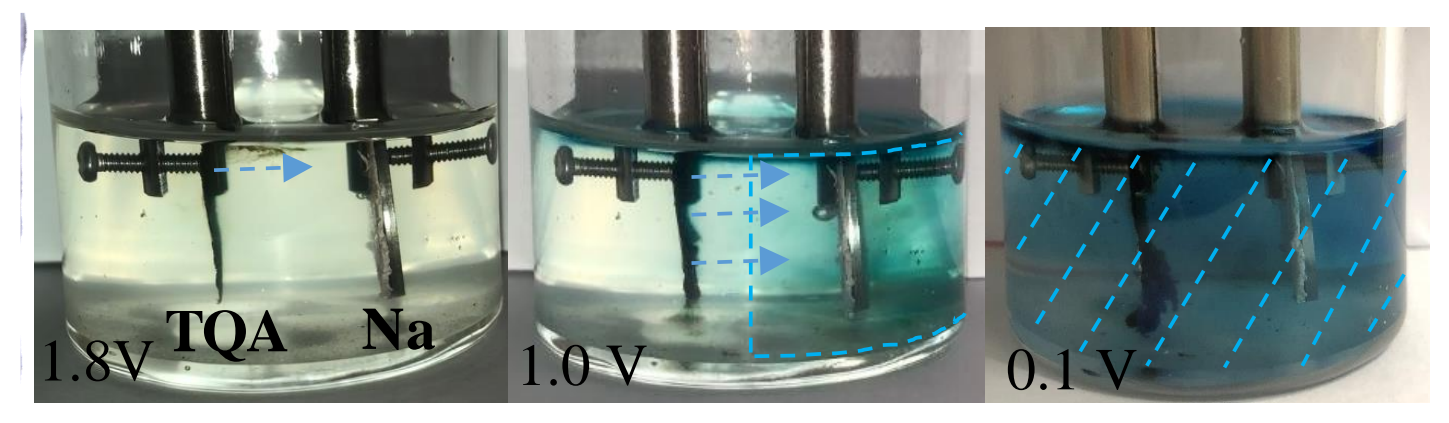

Figure S5. Color change due to dissolution and electro-migration of electrode TQA during discharge electrochemically cycled in a half-cell configuration.

\section{Reference}

(1) Peng, C.; Ning, G.-H.; Su, J.; Zhong, G.; Tang, W.; Tian, B.; Su, C.; Yu, D.; Zu, L.; Yang, J.; Ng, M.-F.; Hu, Y.-S.; Yang, Y.; Armand, M.; Loh, K.P. Reversible Multi-Electron Redox Chemistry of $\pi$-Conjugated N-Containing Heteroaromatic Molecule-based Organic Cathodes. Nature Energy 2017, 2, 17074.

(2) Song, Z.; Zhou, H. Towards Sustainable and Versatile Energy Storage Devices: an Overview of Organic Electrode Materials. Energy Environ. Sci. 2013, 6, 2280-2301. 